\title{
Da necessidade de princípios de Arquitetura da Informação para a Internet das Coisas
}

Flavia Lacerda

Doutoranda (UnB/2011-atual) e mestre em Ciência da Informação. Especialista em Gestão de Tecnologia da Informação

Mamede Lima-Marques

Doutor em Ciência da Computação pela Universite de Toulouse III (Paul Sabatier).Professor do Departamento de Ciência da Informação da Universidade de Brasília.Diretor do Centro de Pesquisa em Arquitetura da Informação (CPAI) da Universidade de Brasília

http://dx.doi.org/10.1590/1981-5344/2356

Analisa-se neste artigo o cenário da Internet das Coisas (IdC) e seus impactos para a sociedade. Identifica-se a necessidade de uma abordagem humanista e sistêmica na área de Arquitetura da Informação, baseada essencialmente em princípios, que componha um arcabouço teórico transdisciplinar para lidar com questões tecnológicas, informacionais e sociais emergentes do fenômeno da IdC.

Palavras-chave: Arquitetura da Informação Pervasiva; Internet das Coisas; Ecossistemas de informação.

\section{On the need for Information Architecture principles to the Internet of Things}

This article analyzes Internet of Things (IoT) scenario and its impacts on society. Identifies the need for a humanistic and systemic approach in Architecture of Information area, essentially based on principles, to compose a transdisciplinary theoretical framework to deal with technological, informational and social issues emerging from IoT phenomenon. 
Keywords: Pervasive Information Architecture; Internet of Things; Information ecosystems.

Recebido em 09.03.2015 Aceito em 06.05.2015

\section{Introdução}

O contexto da sociedade atual aponta para uma realidade de convergência, em que os limites entre concreto e digital se tornam cada vez mais tênues. Na trilogia "A Era da Informação: economia, sociedade e cultura", Castells (1999) faz um amplo diagnóstico da revolução promovida pelas tecnologias da informação e da comunicação, analisando de que forma o advento da Internet redefiniu e continua estabelecendo novas formas de organização da sociedade.

Castells (2003) entende a Internet como "a base tecnológica para a forma organizacional da Era da Informação: a rede". Este fato fica mais evidente na atual geração - caracterizada pela chamada Internet das Coisas (IdC): são sistemas, no sentido amplo, interligados entre si em diferentes escalas, formando ecossistemas com componentes biológicos, materiais, urbanos - tendo em comum a informação como substrato, que passa a fluir e estar presente literalmente em toda parte. Por isso, a IdC é conhecida também como Internet Ubíqua.

$\mathrm{Na}$ IdC, informações passam a se mover em sentido inverso do que ocorria na formação do chamado ciberespaço. O mundo físico é alimentado pelo digital, a realidade é aumentada por aplicações centradas no usuário que promovem consumo e produção de informações. É como se o ciberespaço, que antes era um universo paralelo, transbordasse (KUNIAVSKY, 2010; RESMINI; ROSATI, 2011).

O extraordinário potencial da IdC é o poder que confere aos objetos de uso cotidiano de capturar, processar, armazenar, transmitir e apresentar informações. Interligados em rede, os objetos são capazes de realizar ações de forma independente e gerar dados em quantidade e variedade exponenciais, como produto das interações. Nesse contexto, a informação passa a fazer parte do ambiente, e configuram-se novas formas de atuação das pessoas no mundo.

Considere-se ainda a abrangência, a pervasividade e o crescimento expressivo das aplicações da IdC. A Internet conta atualmente com quase 3 bilhões de usuários conectados, conforme o "Global Internet Report" (KENDE, 2014). A previsão do Gartner (2014) é de que o número de dispositivos conectados seja de 26 bilhões em 2020. Em uma análise mais otimista, a Cisco prevê 50 bilhões de objetos no mesmo período, movimentando um mercado de US\$14,4 trilhões até 2022 (EVANS, 2011). Lucas, Ballay e McManus (2012) anunciam para breve a marca dos trilhões de nós conectados à Rede, número que utilizaram como título do livro dedicado ao tema. Os autores acreditam que a revolução promovida pela IdC representa o futuro da Era da Informação. 
A IdC é compreendida, neste cenário, como um fenômeno complexo, observável a partir de múltiplos pontos de vista - social, cultural, econômico, organizacional, tecnológico, informacional - que tem como consequência direta a materialização de ambientes permeados por informação. O objetivo deste artigo é argumentar sobre a necessidade de uma Arquitetura da Informação baseada em princípios para lidar com as questões emergentes deste fenômeno.

\section{Internet das Coisas (IdC)}

O termo Internet of Things (Internet das Coisas) foi cunhado em 1999 por Kevin Ashton, co-fundador do Auto-ID Center do Massachusetts Institute of Technology (MIT). Em recente artigo, Ashton (2009) afirmou que a ideia original da IdC previa a conexão de todos os objetos físicos à Internet, com capacidade de capturar informações por meio de identificação por radiofrequência (RFID) e tecnologias de sensoriamento as quais os permitiriam observar, identificar e compreender o mundo independentemente das pessoas e suas limitações de tempo, atenção e precisão. Em 2005 a União Internacional de Telecomunicações (UIT) previu que a possibilidade de identificação única de itens, associada a tecnologias de sensores e a capacidade de interagir com o ambiente criaria uma Internet das Coisas (ITU-T, 2012).

Em abordagem didática, Davis (2008) define quatro estágios de evolução da Internet - Web 1.0, voltada para a conexão e obtenção de informações na Rede; Web 2.0 ou Web Social, caracterizada pela preocupação com a experiência do usuário e a colaboração por meio das redes sociais; Web 3.0 ou Web Semântica, com esforços concentrados na atribuição de significado e contexto às informações; e o estágio atual, a Web Ubíqua, constituída pela Internet das Coisas (IdC), fundamentada pela conectividade e interatividade entre pessoas, informações, processos e objetos, por meio de tecnologias que possibilitam acesso à rede por qualquer pessoa, de qualquer lugar, a qualquer tempo, utilizando quaisquer dispositivos, incluindo equipamentos multifuncionais com sensores inteligentes, tais como eletrodomésticos, automóveis, roupas, etc., a partir de aplicações que se adaptam dinamicamente às necessidades dos usuários (DAVIS, 2008; W3C, 2010). Estamos, portanto, na era da computação embutida e distribuída pelo ambiente (KUNIAVSKY, 2010).

\section{Desafios e questões emergentes da IdC}

O mundo físico está se tornando um grande ecossistema de informação. Os objetos tanto podem sentir o ambiente como se comunicar independentemente de intervenções humanas. Tornam-se, portanto, participantes ativos nos processos de negócio, e passam a ser reconhecidos e identificados em ambientes inteligentes, que recuperam dinamicamente informações na Internet, promovendo sua funcionalidade 
adaptativa e responsiva (CHUI; LÖFFLER; ROBERTS, 2010; WEBER, 2013).

A IdC afeta a humanidade em diferentes escalas. Envolve desde nanochips implantados em seres vivos a objetos de uso comum interconectados, equipados com sensores e identificados por RFID capazes de trocar informações entre si, com as pessoas ou com o ambiente - até cidades inteiras sendo projetadas de maneira totalmente conectada e automatizada (as chamadas smart cities ou cidades inteligentes). As formas de manifestação da IdC são heterogêneas, incluindo dispositivos de múltiplos propósitos (celulares, tablets, relógios e óculos inteligentes) e dispositivos especializados (sensores de temperatura, dispositivos ativos e passivos, etc.), suportados por uma variedade de plataformas de software e hardware. O desafio de projetar espaços na IdC é contemplar os diferentes níveis de granularidade de forma transparente, garantindo a interoperabilidade.

As inovações que surgem no âmbito da IdC ampliam o potencial humano em diversas áreas - tais como planejamento urbano (cidades, edifícios e transito inteligentes), meio ambiente (energia, água), indústria, comércio, turismo, educação, saúde, trabalho, segurança, programas sociais, governo - com soluções capazes de promover desenvolvimento econômico, sustentabilidade e qualidade de vida.

O problema é que a velocidade com a qual a tecnologia se difunde na vida das pessoas é maior do que a possibilidade de previsão de seus impactos, sejam positivos ou negativos. E, dada a infinidade de aplicações e a abrangência da IdC, os efeitos negativos são temerários, considerando que o processo de mudança tem sido conduzido em grande medida por empresas privadas, com interesses nem sempre coincidentes com os da sociedade. Lucas, Ballay e McManus (2012), Greenfield e Kim (2013), Greenfield (2006), Norman (2009), e outros autores alertam para os riscos de definições impulsionadas essencialmente por forças de mercado moldarem o futuro.

O urbanista e crítico Adam Greenfield (2006) denomina o fenômeno da IdC de everyware, em alusão aos objetos conectados em toda parte. Ele acredita que a IdC deve ser cuidadosamente arquitetada no momento presente, pois trará cada vez mais implicações para a humanidade. Ressalta que everyware é inevitável, e toda a infraestrutura necessária para sua implantação já existe. Mas a forma que tomará ainda é passível de mudanças e a articulação de padrões essenciais para um desenvolvimento ético e responsável é urgente. A dificuldade está em compreender o significado das escolhas de futuros possíveis em um curto intervalo de tempo, e fazer opções conscientes, considerando os impactos de uma "vida colonizada pela tecnologia da informação". Dependendo de como for definida, everyware pode ser uma questão imediata ou um "problema para cem anos [nas palavras de Gene Becker, da HP]: um desafio técnico, social, ético e político de extraordinária sutileza e dificuldade [...]. Mapear as necessidades e vontades de pessoas reais em qualquer situação está longe de ser tarefa óbvia" (GREENFIELD, 2006, p. 179). 
A questão do poder de atuação (ou agência) conferida aos objetos na IdC é um dos pontos mais críticos para discussão. Objetos tornam-se ativos, capazes de promover ações independentemente dos seres humanos. Santucci (2014) preocupa-se com a autonomia das pessoas em um mundo onde os objetos conectados inteligentes superam os humanos em uma proporção de pelo menos 1 a 10. Como observa Floridi (2013, min. 1:14), as pessoas podem simplesmente não querer interagir com entidades-robôs. "Quem vai se adaptar a quem?", afirma.

Objetos ganham vida, passam a ser agentes, a perceber estímulos do ambiente, produzir informações e interagir com as pessoas e outros objetos. Mas a responsividade e a interatividade foram concebidas a partir de premissas, baseadas no modelo mental e nas motivações de quem as idealizou. É preciso investigar até que ponto as respostas inteligentes e decisões independentes dos objetos estão de acordo com as necessidades e vontades dos seres humanos que os utilizam. Um dos perigos apontados por Donald Norman (2009) é o de pessoas criativas e produtivas tornarem-se servos dos objetos, preocupando-se mais com seu funcionamento do que usufruindo de seus benefícios.

"Como mudar a forma de interação com nossas máquinas para obter melhores vantagens de suas virtudes e forças, ao mesmo tempo eliminando as ações inoportunas e perigosas?", indaga Norman (2009, p. 8). A melhor maneira de promover a interação suave entre pessoas e dispositivos inteligentes é aumentando a coordenação e a cooperação entre ambos, sugere o autor. Afirma ainda que os produtos estão ficando mais espertos, inteligentes, exigentes e presunçosos. Passam a fazer parte de um ecossistema social, portanto, precisam de traquejo, habilidades comunicativas, e até mesmo emoções. O autor ressalta a dificuldade de ignorar um sistema que não conseguiu antecipar a necessidade de um usuário: "ele pode simplesmente ignorá-lo, se for possível ignorar uma casa que constantemente pisca sugestões sobre contadores, paredes e pisos". McEwen e Cassimally (2013, p. 24) observam que este problema ganha dimensões mais amplas ao incluíremse as centenas de novos serviços e aplicações espalhadas pelos objetos ao redor do mundo: "haverá uma cacofonia em busca de atenção".

"Como pode uma máquina discernir o que é importante, se os contextos de importância variam?", continua Norman (2009, p. 11). Para - autor, a falta de senso comum é a maior causa da inabilidade de comunicação entre pessoas e máquinas. O senso comum das máquinas é a medida do senso comum daqueles que as programaram, em um momento desprovido de contexto. O contexto é apenas inferido. Quando isso ocorre com uma pessoa, é esperado que utilize criatividade para a solução de problemas". Nesse sentido, o autor indaga: "seriam estes sistemas realmente inteligentes" e afirma: "não, são apenas responsivos" (NORMAN, 2009, p. 29). A inteligência está na mente daqueles que os projetaram, e que cuidadosamente tentam antecipar todas as condições e reações possíveis em cada situação, para programar respostas adequadas. Nessa linha, Floridi (2013, min. 00:43) concorda que os dispositivos sejam 
espertos (smart) - capazes de fazer coisas interessantes; mas não inteligentes.

Em geral, defende Norman (2009), as soluções são valiosas e úteis, mas ocorrem falhas, pois raramente é possível inferir o conjunto de informações contextuais e circunstanciais com a mesma acurácia e riqueza dos sensores próprios dos seres humanos, que além de tudo criam representações complexas do mundo e de suas ações, bem como expectativas precisas com base em uma longa história de interação. Ele acredita que o risco não significa que o melhor caminho seja "rejeitar a ajuda das máquinas inteligentes, pois podem ser verdadeiramente proveitosas; mas é preciso socializá-las, para que aprimorem a forma com a qual se comunicam e interagem com as pessoas".

A questão da agência dos objetos traz ainda dificuldades na determinação da responsabilidade sobre os atos, notadamente em situações imprevistas que gerem dano, prejuízo ou consequências indesejáveis. É preciso haver uma espécie de contrato social entre pessoas e objetos, com as respectivas ramificações éticas. Em um exemplo concreto: deve haver maior preocupação com as bases éticas e consequências de delegar decisões para sistemas automotivos do que com as questões tecnológicas dos carros inteligentes (EUROPEAN COMMISSION, 2012). No caso de um acidente provocado pela ação de um automóvel smart, por exemplo, quem seria responsabilizado?

Em uma perspectiva mais ampla, Greenfield e Kim (2013, p. 519) questionam: "estariam os arquitetos das cidades inteligentes suscetíveis a responsabilização (accountability) democrática, considerando o nível de interferência que podem exercer na vida das pessoas no contexto atual?". Decisões de arquitetos são atos políticos em âmbito urbano - do mesmo modo como a autoria de um algoritmo destinado a promover a distribuição de recursos cívicos em uma cidade.

Outro ponto é o problema do consentimento informado. Greenfield (2006, p. 66) alerta: "as pessoas podem entrar em everyware de forma inadvertida, inconsciente ou indesejada. A natureza passiva da exposição dos indivíduos a essa infraestrutura de rede pervasiva e os métodos de coleta de dados têm implicações sobre sua vida, saibam ou não, queiram ou não". Nesses ambientes, a informação está sempre sendo coletada e utilizada como base para ação, arquivada e recuperada de maneira sutil. Os equipamentos operam em conjunto para produzir significado - mas "de que forma a parafernália tecnológica universal se adapta a práticas, atividades, leis e hábitos locais?", questiona (GREENFIELD, 2006, p. 29).

Greenfield (2006) indaga se as aplicações ubíquas cumprirão a promessa de uma "tecnologia calma" - conforme imaginado por Weiser e Brown (1996) - onde a sobrecarga cognitiva envolvida nas interações entre pessoas e objetos é reduzida, resultando em interfaces compreensíveis, úteis e usáveis. Ele acredita que as atuais práticas de desenvolvimento em tecnologia da informação (TI) aplicadas a everyware resultarão em experiências de uso inaceitavelmente ruins caso não sejam repensadas; e lembra que o design adequado para everyware é 
infinitamente mais complexo do que o design de websites ou aplicações desktop.

Em relatório do Gartner (2013), a IdC foi apontada como uma das dez tendências estratégicas mundiais de tecnologia de prestação de serviços eletrônicos. O tema tem sido tratado como prioritário pelo setor público de diversos países, especialmente os europeus e asiáticos, com programas de governo dedicados a identificar impactos e potenciais oportunidades trazidas pelas inovações. Nessa conjuntura, a preocupação com a governança da Internet, traduzida na capacidade de definir modelos de funcionamento da rede, torna-se fundamental para garantir seu desenvolvimento sustentável e o atendimento pleno aos interesses da sociedade.

Tecnologias e mercados não podem existir independentemente de princípios abrangentes de um sistema ético e social. A IdC terá um amplo impacto em muitos dos processos que caracterizam a vida cotidiana, portanto, é primordial que seu desenvolvimento seja fundamentado em estratégias orientadas a pessoas. Para isso, aqueles que as projetam devem estar próximos aos que irão utilizá-las (MENDES, 2011).

Numa das palestras de abertura do evento Internet of Things: Philosophy, que teve como mote examinar o que significa ser humano na Internet das Coisas, Gérald Santucci (2014) indagou: como a Internet das Coisas irá transformar-nos - e em quê? Segundo ele, se tivéssemos de escolher uma palavra para descrever o que é a IdC é, certamente seria inteligente: objetos, cidades, redes, saúde e transporte inteligentes, etc. Mas o que inteligente implica em termos de desafios sociais? Quais as formas de governança necessárias em um ambiente inteligente? Como a IdC afeta e transforma a relação entre seres humanos e objetos? Que ética deve orientar a concepção e implementação de objetos inteligentes conectados? Não seriam objetos forçando os seres humanos a se comportarem de acordo com padrões que podem privá-los de sua autonomia ou liberdade?

O grupo de especialistas da Comissão Europeia (IoT Expert Group) identificou seis questões éticas fundamentais no âmbito da IdC: justiça social (combate à exclusão digital e de conhecimentos); confiabilidade (garantia de privacidade e segurança, proteção de dados); clareza sobre contextos (responsabilidades dos atores no ecossistema, privado versus público), clareza sobre metáforas (conveniência versus perigos das coisas inteligentes), clareza sobre agência de objetos (contrato entre pessoas e objetos) e autonomia dos indivíduos (consentimento informado sobre funcionalidades e ações dos objetos) (EUROPEAN COMMISSION, 2012). Estamos caminhando para "um mundo rico em informações, com novas possibilidades e problemas. Indivíduos e instituições obterão maior flexibilidade e produtividade. No entanto, teremos que lutar para equilibrar privacidade, liberdade, conveniência e segurança" (MORVILLE, 2005, p. 3). 


\section{Princípios de Arquitetura da Informação para a IdC}

Informações estão sendo incorporadas em objetos de uso comum em toda parte. Isto muda fundamentalmente a maneira de compreender a Arquitetura da Informação, a forma de lidar com suas questões científicas e, definitivamente, a forma de praticá-la (LACERDA; LIMAMARQUES, 2014, p.7).

Resmini e Rosati (2011, p. 681) preveem a "necessária adoção de uma visão abrangente para o design de espaços de informação" nessa realidade de convergência física e digital. No prefácio da obra de Resmini e Rosati (2011, p. 97), Peter Morville questiona: "como responder aos novos desafios de criação de caminhos e lugares que conectem espaços físicos, digitais e cognitivos?"

Em Digital Ground, McCullough (2004) parte de princípios arquitetônicos para abordar a modelagem de espaços onde ocorrem ações mediadas digitalmente. Observa que a computação pervasiva cria tais espaços, que subitamente reconfiguram-se de acordo com seus ocupantes, cujo uso pode causar paranoia ou satisfação, dependendo de quão inteligentemente são projetados. E coloca em pauta questões do tipo: "é preferível ser sujeito passivo de monitoramento ou precisar fornecer ativamente identificação?". "Arquiteturas instanciam intenções, etiquetas e ações particulares", conclui (MCCULLOUGH, 2004, p. 47).

A conferência "Internet of Things: Philosophy", ocorrida em 2014, propôs um debate sobre as ramificações da IdC em um contexto filosófico. Dentre as questões levantadas no evento estão: influência da tecnologia sobre as emoções; auto-percepção do ser humano e do modo como se relaciona com as coisas; aumento do potencial humano de ação e criação pela tecnologia; mudanças na forma de comunicação, e significado da comunicação homem-máquina e máquina-máquina.

A comunidade de Arquitetura da Informação está se movendo para a expansão das visões de mundo, de seu escopo de ação e de suas motivações, como demonstram os discursos de Morville (2012), Arango (2012), Resmini (2013), Hinton (2013), e Klyn (2013), entre outros. As discussões estão sendo realizadas em publicações, redes sociais e eventos, como as edições do IA Summit 2013, em Baltimore (EUA) e 2014 em San Diego, onde ocorreram mesas redondas sobre o tema Reframing Information Architecture (Reenquadrando a Arquitetura da Informação) (LACERDA; LIMA-MARQUES, 2014).

Chamar para uma renovação epistemológica significa pedir aos estudiosos da disciplina para elevarem-se ao meta-nível do sistema de investigação, a fim de questionar algumas das abordagens atuais. A produção de trabalho inovador duradouro sinaliza o advento de um novo paradigma, ou, pelo 
menos, a modificação do existente (VAN GIGCH, 1990, p. 127).

No livro "Pervasive Information Architecture", Resmini e Rosati (2011) propõem o conceito de Arquitetura da Informação Pervasiva como uma abordagem voltada às questões relativas ao design de ecossistemas de informação em ecologias ubíquas. Pela definição dos autores:

Nós denominamos esses novos espaços extendidos de informação - nos quais interagimos tanto com entidades digitais quanto físicas - ecologias ubíquas: são sistemas que conectam pessoas, informações, processos, que estão em toda parte. São arquiteturas da informação pervasivas. São a camada estruturante que atravessa as diferentes mídias, canais e processos: na qual expressamos nosso eu expandido, socialmente (RESMINI; ROSATI, 2011, p. 140).

Lucas, Ballay e McManus (2012, p. 171) buscam em sua obra identificar padrões de design e processos que possam ser utilizados na intersecção entre sistemas de informação e pessoas. O que eles chamam de "rede de trilhões de nós" irá requerer "a emergência de um estilo de Arquitetura da Informação". "Em uma camada acima da arquitetura de sistemas (que trata de como os computadores em si são construídos), e abaixo da camada de interface de usuários (que é sobre como os sistemas se comunicam com usuários), a Arquitetura da Informação trata do design da informação em si. A rede de trilhões implica em um vasto e heterogêneo fluxo de informações. O único ponto em comum desse fluxo é a informação, e é onde devemos concentrar esforços de design se quisermos buscar integridade global".

Hiroshi Ishii (2012), cientista e professor do Massachusetts Institute of Technology (MIT), acredita que a inovação tecnológica deve ser direcionada por uma visão (conceitos, princípios) que fundamente o desenvolvimento de aplicações para atender a necessidades de usuários, materializando-se em tecnologias (Figura 1). Proporcionalmente, e considerando a atual conjuntura, as tecnologias teriam a vida útil estimada em um ano, as aplicações atenderiam necessidades de usuários por aproximadamente dez anos, e os princípios perdurariam por mais de cem anos (Figura 2). Segundo Ishii (2012, p. 50), "princípios raramente mudam, mas práticas sempre dependem de contexto. Ao associar um método a uma coleção de princípios, é sempre possível criar novas práticas, desde que aderentes aos princípios". 
Figura 1 - Fatores de inovação tecnológica

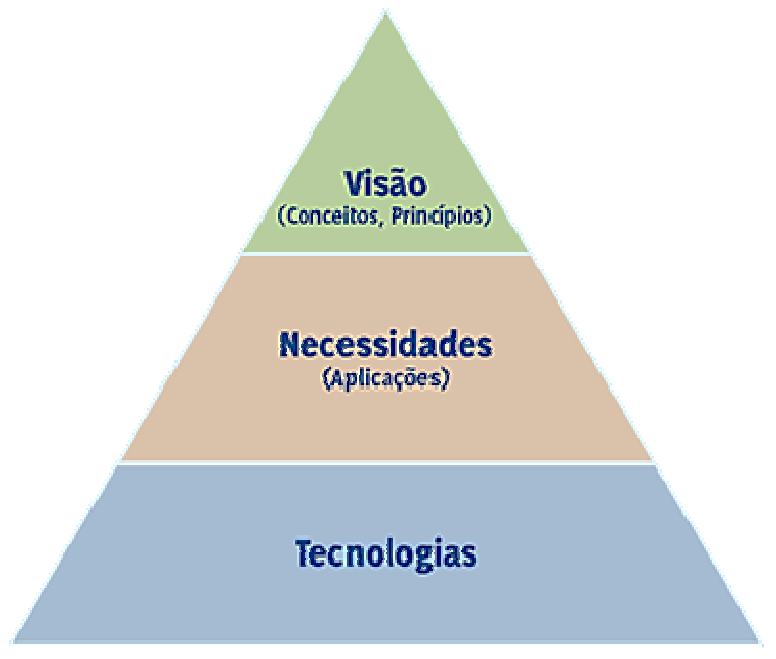

Fonte: ISHII (2012).
Figura 2 - Vida útil dos fatores de inovação

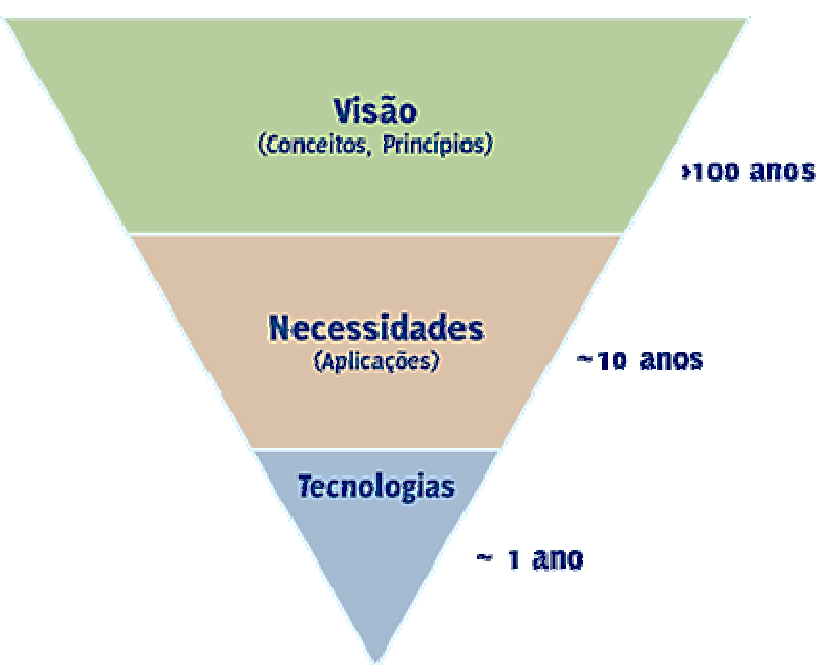

Fonte: ISHII (2012).

Greenfield (2006, p. 257) defende que um conjunto explícito de princípios será extremamente útil tanto para desenvolvedores quanto para usuários em everyware. E alerta que "os princípios são necessários, mas não suficientes: constituem não um fim, mas um começo". Há que se construir um everyware que se adapte às pessoas, não o contrário. Mas não será trivial projetar sistemas ubíquos sofisticados o suficiente para capturar a riqueza de nuances da vida cotidiana (GREENFIELD, 2006).

Diretrizes serão claramente úteis para quem está adquirindo ou utilizando everyware, ainda que limitadas por serem contingentes, provisionais e incompletas. Se houver um conjunto de critérios compacto, objetivo, e amplamente acordado, haverá compatibilidade e interoperabilidade. Os princípios focam não em como alcançar uma quantidade de finalidades, mas em que finalidades devem ser perseguidas prioritariamente. É preciso desenvolver um arcabouço de conhecimentos, padrões e convenções, começando pelas interfaces pelas quais acessaremos everyware (GREENFIELD, 2006).

Desde que a Arquitetura da Informação é baseada em princípios que são amplamente independentes de qualquer mídia específica - afinal diz respeito à estruturação de espaços de informação tanto quanto a Arquitetura preocupa-se com a estruturação de espaços físicos - [a área] fornece um modelo conceitual flexível, porém sólido, para o design de esperiências em múltiplos canais [cross-channel] e múltplos contextos [cross-context], que se extendem a diversas mídias e ambientes (RESMINI; ROSATI, 2011, p. 1369). 
Shin (2010) destaca que ainda são poucos os esforços de pesquisa concentrados nas imensas repercussões sociais, culturais e comportamentais da IdC, que tenham a finalidade de promover sua implantação, gestão e evolução na qualidade de inovação sócio-técnica (BIJKER, 1997), que deve ser projetada e desenvolvida como um sistema centrado nos humanos. Lucas, Ballay e McManus (2012) projetos de sistemas de informação para a IdC devem considerar: métodos profundamente interdisciplinares; foco humanista; design de interação centrado em informações; e computação em contexto.

\section{Conclusão}

O cenário da IdC é multifacetado e vem sendo tratado na literatura sob perspectivas socioculturais, econômicas, filosóficas, mas predominantemente tecnológicas. As questões emergentes deste contexto são de natureza e proporções variadas - como privacidade, usabilidade, consentimento, para citar alguns - e afetam desde o domínio individual até o global, passando pelas esferas doméstica, social, urbanística, governamental.

A pesquisa voltada para aspectos tecnológicos é fundamental para o avanço da IdC. Entretanto, é necessário compreender os tipos de tarefas e padrões de interatividade que emergem no momento em que o usuário transcende o modelo de interação com computadores para interagir com interfaces que permeiam seu ambiente e aumentam suas capacidades individuais, mas que, ao mesmo tempo, conferem autonomia e poder de decisão aos objetos.

Há uma série de desafios tecnológicos e informacionais que devem ser tratados para viabilizar o funcionamento adequado da IdC. Mas está nos aspectos humanos e sociais a oportunidade de fazer a diferença em termos de projetos de ecossistemas de informação direcionados às necessidades das pessoas.

A questão central de interesse da Internet das Coisas para a Arquitetura da Informação, na qualidade de disciplina da Ciência da Informação, é a concretização de um mundo onde o processamento de informações estará em toda parte. São novos espaços de informação, com diferentes propriedades, que precisam ser compreendidas e arquitetadas. A Internet das Coisas requer de uma abordagem humanista e sistêmica, baseada essencialmente em princípios, com vistas a evitar soluções pautadas em valores comerciais ou tecnicistas. Indica-se, nesse sentido, a necessidade de estruturação de um arcabouço teórico transdisciplinar no âmbito da área de Arquitetura da Informação para lidar com as questões emergentes da IdC.

\section{Referências}

ARANGO, J. World information architecture day 2012. . [S.I: s.n.], 2012. Disponível em: 
<http://www.youtube.com/watch?v=kY5CC2QfevE\&feature=youtube_gda ta_player>. Acesso em: 15 set. 2013.

ASHTON, K. That "Internet of Things" Thing. RFID Journal, 22 jun. 2009. Disponível em: <http://www.rfidjournal.com/articles/view?4986>. Acesso em: 31 ago. 2013.

BIJKER, W. E. Of bicycles, bakelites, and bulbs: toward a theory of sociotechnical change. Cambridge, Mass.: MIT Press, 1997.

CASTELLS, M. A galáxia Internet: reflexões sobre a Internet, negócios e a sociedade. Rio de Janeiro: Zahar, 2003.

CASTELLS, M. A sociedade em rede. São Paulo: Paz e Terra, 1999. v. 1. (A Era da Informação: economia, sociedade e cultura).

CHUI, M.; LÖFFLER, M.; ROBERTS, R. The internet of things. McKinsey Quarterly, V. 2, 2010. Disponível em: <http://scholar.google.com.br/scholar?cluster $=4109556744406282630 \& \mathrm{~h}$ I=pt-BR\&as_sdt=0,5>. Acesso em: 3 jan. 2013.

DAVIS, M. Semantic wave 2008 report: industry roadmap to Web 3.0 \& Multibillion Dollar Market Opportunities. Executive Summary, 2008. Disponível em: <http://www.project10x.com>. Acesso em: 20 jul. 2010.

EUROPEAN COMMISSION. Conclusions of the Internet of Things public consultation. , $\mathrm{n}^{\circ}$ 9. Brussels: [s.n.], 12 abr. 2012. Disponível em: $<$ ec.europa.eu//digital-agenda/en/news/conclusions-internet-thingspublic-consultation>. Acesso em: 1 jan. 2015.

EVANS, D. The internet of things: how the next evolution of the internet is changing everything. CISCO white paper, 2011. $11 \mathrm{p}$.

FLORIDI, L. Internet of Things: atart of a revolution. [S.I: s.n.], 2013. Disponível em: <http://vimeo.com/77209992>. Acesso em: 14 jun. 2014.

GARTNER. Gartner identifies the top 10 strategic technology trends for 2013. [S.I: S.n.], mar. 2013. Disponível em: <http://www.gartner.com/it/page.jsp?id=2209615>. Acesso em: 1 jan. 2013.

GARTNER. Gartner Says the Internet of Things installed base will grow to 26 billion units by 2020. Disponível em: <http://www.gartner.com/newsroom/id/2636073>. Acesso em: 28 jul. 2014.

GREENFIELD, A. Everyware: the dawning age of ubiquitous computing. San Francisco: New Riders Publishing, 2006.

GREENFIELD, A.; KIM, N. Against the smart city: the city is here for you to use. [S.I.]: Do projects, 2013.

HINTON, A. A model for information environments: reframe IA. In: IA SUMMIT, abr. 2013, Baltimore, MD, USA. Anais... Baltimore, MD, USA: [s.n.], abr. 2013. Disponível em: 
<http://www.slideshare.net/andrewhinton/a-model-for-informationenvironments-reframe-ia-workshop-2013>. Acesso em: 15 set. 2013.

INTERNET OF THINGS: Philosophy. 3 jul. 2014, York St John University, UK. Anais... York St John University, UK: [s.n.], 3 jul. 2014. Disponível em: <http://internetofthingsphilosophy.com/slides-proceedings-etc/>. Acesso em: 1 ago. 2014.

ISHII, $\mathrm{H}$. Defy gravity: beyond tangible bits, toward radical atoms. In: DW/AD '12, 4 dez. 2012, Kyoto International Conference Center, Kyoto, Japan. Anais... Kyoto International Conference Center, Kyoto, Japan: [s.n.], 4 dez. 2012. Disponível em: <http://www.youtube.com/watch?v=SMdFus2nPyw\&feature=youtube_gd ata_player>. Acesso em: 20 abr. 2014.

TELECOMMUNICATION STANDARDIZATION SECTOR (ITU-T). ITU-T Y.2060: overview of the internet of things. . [S.I: s.n.]. Disponível em: <http://www.itu.int/ITU-T/recommendations/rec.aspx?rec $=11559>$. Acesso em: 20 set. 2014.

KENDE, M. Global Internet report. Geneva: Internet Society, 2014. Disponível em: <http://www.internetsociety.org/doc/global-internetreport>. Acesso em: 28 jul. 2014.

KLYN, D. Dutch uncles, ducks and decorated sheds: reframing IA. Baltimore, MD, USA: [s.n.], 2013. Disponível em: <http://www.slideshare.net/danfnord/dutch-uncles-ducks-and-decoratedsheds-reframing-ia>. Acesso em: 18 set. 2013.

KUNIAVSKY, M. Smart things: ubiquitous computing user experience design: ubiquitous computing user experience design. [S.I.]: Elsevier, 2010.

LACERDA, F.; LIMA-MARQUES, M. Information architecture as a discipline: a methodological approach. In: RESMINI, A. (Org.). Reframing Information Architecture. Human-Computer Interaction Series. Switzerland: $\quad$ Springer, $2014 . \quad$ Disponível em: <http://www.springer.com/computer/hci/book/978-3-319-06491-8>.

Acesso em: 19 abr. 2014.

LUCAS, P.; BALLAY, J.; MCMANUS, M. Trillions thriving in the emerging information ecology. Hoboken, N.J: Wiley, 2012.

McCULLOUGH, M. Digital ground: architecture, pervasive computing, and environmental knowing. Cambridge, Mass.: MIT Press, 2004.

McEWEN, A.; CASSIMALLY, H. Designing the Internet of Things. 1 ed. Chichester: Wiley, 2013.

MENDES, P. Social-driven internet of connected objects. [S.I.]: Citeseer, 2011.

MORVILLE, P. Ambient findability: what we find changes who we become. Sebastopol: O'Reilly Media, 2005. 
MORVILLE, P. Ubiquitous information architecture: cross-channel strategy. [S.I: $\quad$ s.n.], 2012. Disponível em: <http://www.slideshare.net/morville/ubiquitous-information-architecturecrosschannel-strategy>. Acesso em: $30 \mathrm{dez} .2012$.

NORMAN, D. The design of future things. New York: Basic Books, 2009.

RESMINI, A. The poetics of information architecture. In: ASIS\&T EUROIA, 2013, Edinburgh. Anais... Edinburgh: [s.n.], 2013. Disponível em: <http://pt.slideshare.net/resmini/the-poetics-of-information-architecture26991604>. Acesso em: 3 ago. 2014.

RESMINI, A.; ROSATI, L. Pervasive information architecture: designing cross-channel user experiences. Boston: Morgan Kaufmann, 2011.

SANTUCCI, G. On the philosophy of the Internet of Things. In: INTERNET OF THINGS PHILOSOPHY, 3 jul. 2014, York St John University. Anais... York St John University: [s.n.], 3 jul. 2014. Disponível em: <http://www.theinternetofthings.eu/content/g\%C3\%A9rald-santucciinternet-things-window-our-future $>$. Acesso em: 2 fev. 2015.

SHIN, D.-H. A realization of pervasive computing: Ubiquitous city. In: TECHNOLOGY MANAGEMENT FOR GLOBAL ECONOMIC GROWTH (PICMET), 2010 PROCEEDINGS OF PICMET '10: jul. 2010. Proceedings... [S.I: s.n.], jul. 2010. p. $1-10$.

VAN GIGCH, J. P. Systems science, the discipline of epistemological domains, contributes to the design of the intelligent global web. Behavioral Science, v. 35, n. 2, p. 122, 1990. Disponível em: <http://onlinelibrary.wiley.com/doi/10.1002/bs.3830350205/abstract>. Acesso em: 20 jul. 2010.

W3C. Ubiquitous Web Domain. W3C. [S.I: s.n.], 2010. Disponível em: <http://www.w3.org/UbiWeb/>. Acesso em: 20 jul. 2010

WEBER, R. H. Internet of things: governance quo vadis? Computer Law \& Security Review, v. 29, n. 4, p. 341-347, ago. 2013. Disponível em: <http://www.sciencedirect.com/science/article/pii/S0267364913001015>. Acesso em: 28 ago. 2013.

WEISER, M.; BROWN, J. S. The coming age of calm technology. Palo Alto: Xerox PARC, 1996. 